the Cavendish Chair of Physics at the University of Leeds which he has held since 1952. His research in cosmic rays started under C. T. R. Wilson and he became a prominent member of P. M. S. Blackett's group at Manchester after the Second World War. Latterly he has been responsible for setting up and developing the EAS detector array at Haverah Park. He plans to continue his interest in the field during his retirement.

J. G. Wilson presented a paper dealing with the energy spectrum of cosmic rays at energies $\gtrsim 10^{17} \mathrm{eV}$. Evidence continues to mount in support of a flattening of the spectrum above $10^{19}$ $\mathrm{eV}$. There appears to be no cut-off at this energy arising, as predicted, from interaction with the supposed universal $3 \mathrm{~K}$ photon flux. This inconsistency still remains a problem as the arrival direction analysis does seem to suggest an extragalactic origin for these very high energy cosmic rays.

In general then cosmic ray research still appears to be a hive of activity with plenty of promise. However, progress is inevitably slow, perhaps reflecting the difficulties of both technique and interpretation.

\section{Efficient bumblebees are almost specialists}

\section{from John Krebs}

THE familiar sight of bumblebees gathering pollen and nectar from wild flowers is the basis of a recent study by B. Heinrich (Ecol. Monogr., 46, 105; 1976), in which he analysed the foraging behaviour of individually marked worker bees of the genus Bombus. The bee uses different techniques to collect pollen and nectar from different plant species, for example it collects pollen from dogwood by pressing its body on the flat inflorescence, while on the cranberry it hangs upside down and shakes the pollen onto its body. The bees in Heinrich's study area preferred certain flower species over others: Chelone being the most favoured and Solidago the least. In fact the rank order of preference (measured by the number of bees per flower) corresponded exactly to the rank order of potential nectar yield from the various species-I will return to this point later. While this picture applies to the whole bee population, each individual bee specialises on a particular plant species (its 'major') and occasionally visits one or two other species ('minors'): the overall distribution of foraging effort by the bees is the sum of many specialists on different plants.
Specialisation is not purely a result of attachment to one particular site, nor is it temporary; many workers probably retain the same 'major' throughout their life of four to six weeks. The 'minors' of individual bees are also constant, but Heinrich found that he could induce workers to adjust their preference by adding sugar droplets to a 'minor' in their repertoire, which then became the 'major', showing that the choice of a 'major' is related to how profitable different plants are. The benefit to a bee of specialising was revealed in an experiment in which Heinrich compared the efficiency of experienced and naive bees in extracting nectar from monkshood, which is unusual in having its nectar hidden in two modified petals. The naive bees, which had foraged on other flowers but not monkshood, had difficulty in finding and extracting the nectar while the specialists did not. This and other observations suggested that a non-specialist bee would be rather unsuccessful in competing for nectar and pollen with a specialist.

Heinrich's results pose two intriguing questions: Why do all bees not major on the most profitable flower species? And why do individuals maintain minors in their repertoire? The answer to the first question is straightforward. As I have mentioned, the bee population as a whole distributed its foraging effort according to the order of profitability of the various flowers, so that the commonest major is the top ranking nectar source, the next commonest major the second ranking plant and so on. It also turns out that after the foragers have exploited an area, all the different plants contain about the same amount of left-over nectar-they are all reduced to the same level of profitability. This means that the lower nectar content of poor quality flowers is (more or less) exactly compensated for by the fact that more bees compete for nectar in the rich flowers. (This pattern of harvesting, which has also been observed in birds, is predicted by theoretical models describing how a maximally efficient forager ought to behave, but more of that another time.)

The question of why bees maintain minors is more difficult, but G. Oster and B. Heinrich (Ecol. Monogr., 46, 129 ; 1976) have developed a model to offer a tentative explanation. Their argument goes like this: if there is an array of flowers giving different quantities of nectar per unit effort, an efficient worker with perfect knowledge should forage purely in the most profitable flowers. When the bee has no previous knowledge, it has to sample the flowers to estimate their profitabilities and then adopt the "pure strategy" of foraging in the best flowers. But suppose Nature is awkward, and the different flower types continually change ranks, what should the bee do? In the extreme case, when Nature obeys Murphy's Law and what the bee thinks is best immediately becomes the worst, a "mixed strategy" is best because it insures against a sudden change. Assuming that Nature is perverse but not too perverse, the bee's best strategy lies somewhere between mixed and pure: majoring with one or two minors.

\section{Photobiology}

from R. P. F. Gregory

The Seventh International Congress on Photobiology was held in Rome on August 25-September 3, 1976.

WHAT advances stand out after four years' study of photobiology? G. Porter (Royal Institution, London) gave one answer in terms of picosecond kinetics. The primary processes of lightabsorption by a pigment and its reemission in fluorescence can now be studied at times approaching a few picoseconds using the streak camera. (The absolute theoretical barrier was set by the Uncertainty Principle in the region of $0.5 \mathrm{ps}$.) Both by advances in the understanding of the behaviour of excited states of pigments and the application of ultrashort pulsed laser spectroscopy, models have been produced of the operation at the nanosecond timescale of three photobiological pigments. M. Ottolenghi (Hebrew University, Jerusalem) described the interconversions of rhodopsin, bathorhodopsin and isorhodopsin in terms of the energy level diagram and was able to account for the observed quantum yield. Remarkably similar behaviour was reported for the analogous pigment bacteriorhodopsin from the typical phototroph Halobacterium (R. H. Lozier and colleagues, Ames Research Center, California). The phototransformations of phytochrome, preceding the appearance of the $\mathrm{Pr}$ and Pfr forms, are also in the model stage (R. E. Kendrick, University of Newcastle-upon-Tyne). Outside the formal sessions it was apparent that the problems of pigment organisation and the spectroscopic methods presently available for their investigation were common to these topics and also to the study of chlorophyll, both in membranes and in pigment-protein complexes. At this level Photobiology very clearly exists as a discipline. 